\title{
BJMHR
}

British Journal of Medical and Health Research

Journal home page: www.bjmhr.com

\section{The Incidence of Decolonizing Patients of Staphylococcus Aureus Nasal Carriage Undergoing Breast Cancer Surgery In The Netherlands.}

\author{
Gerda $^{1} *$, Geertruida Martina ${ }^{1}$, Anneke $^{2}$, Peter $^{3}$ \\ 1. Margaretha Lelieveld-Vroom, Alrijne hospital, Department of Infection Control. \\ 2. Anneke, Anna Maria Zeillemaker, M.D, Alrijne hospital, Department of Surgery. \\ 3. Peter, Alexander Neijenhuis, M.D, Alrijne hospital, Department of Surgery.
}

\section{ABSTRACT}

Staphylococcus aureus (S. aureus) is the leading nosocomial (hospital acquired) pathogen in hospitals globally, resulting in substantial morbidity, mortality and additional costs. Breast cancer wound infection can delay and even cause the omission of necessary adjuvant oncological treatment. Nasal carriers of S. aureus are a well defined risk factor for subsequent infections with this organism. Decolonization of nasal and extra nasal sites preoperative can reduce the risk of surgical site infections (SSIs) with S. aureus. The aim of this study was to evaluate the effect of patients screened with S. aureus nasal carriage on the incidence of SSI in a breast cancer surgery population. A prospective cohort study was performed between April 2009 and December 2016 including all patients undergoing a breast cancer surgery. Patients were screened for $\mathrm{S}$. aureus nasal carriage and, when tested positive, were subsequently treated with mupirocin nasal ointment and chlorhexidine soap. The control group was a cohort of patients from April 2009 till July 2011, who were not screened and not received treatment. A total of 1543 patients were included in this study. The rate of S. aureus infection was $1.1 \%$ (12 of 1071) for patients who were screened for S. aureus nasal carriage as compared with $2.5 \%$ (12 of 472) for patients who were not screened for S. aureus carriage (relative risk of infection, $0.48 ; 95 \%$ confidence interval $[\mathrm{CI}], 0.194$ to $0.974 ; \mathrm{p}=0.038$ ). The number of surgical site for patients infected with S. aureus who were also operated for breast cancer can be significantly reduced by screening and decolonizing nasal carriers of

S. aureus on admission.

Keywords: Screening, Treatment, Nasal carriage 


\section{INTRODUCTION}

In the Netherlands, breast cancer is the second leading cause of death in women. ${ }^{1}$ Although breast cancer treatment entails a clean surgical procedure, the rate of breast surgical site infections (SSI) range from 0 to 9.6 percent in the Netherlands, depending on definition of SSI, type of operation, comorbidities of the patients, time of follow up, perioperative therapy and reporting institution as described in the PREZIES protocol. ${ }^{2}$ PREZIES is a national nosocomial infection surveillance system in the Netherlands which records SSI data.

SSI of the breast do not only result to longer hospital stay and increased hospital cost, it also leads to delay and omission of necessary adjuvant oncological treatment and psychological trauma. ${ }^{3-4}$ SSI contamination occurs probably during the perioperative phase, with the main sources of microorganisms being the patients gastro-intestinal and the respiratory tracts and skin (endogenous). Staphylococcus aureus (S. aureus) nasal carriage is associated with an increased risk of developing a health care-related infection with this micro-organism. ${ }^{5}$ The risk of infection in nasal carriers is estimated to be two to twelve times higher than in those who are not colonized with $S$. aureus. ${ }^{6}$

Little is known about the results of treatment of nasal carriage caused by breast cancer surgery, despite S. aureus being reported as the leading cause of SSI during breast cancer surgery in the Netherlands. ${ }^{7}$ The objective of this study is to minimize S. aureus nasal carriage related SSIs through the screening for $S$. aureus of patients with breast cancer.

\section{MATERIALS AND METHOD}

\section{Study population}

A prospective cohort study was performed from April $1^{\text {st }} 2009$ until December $31^{\text {st }} 2016$ at the Department of Surgery, Alrijne Hospital, Leiderdorp, Netherlands. A total of 1543 patients operated for breast cancer were included in this study. The surgical procedures included mamma ablation and mamma lumpectomy. Exclusion and inclusion criteria were defined in agreement with PREZIES protocol. ${ }^{2}$

Screening patients on $S$. aureus nasal carriage started from July $1^{\text {st }}, 2011$. In the remaining parts of this study, this group of patients would be referred as the intervention group. From April 2009 until July 2011, patients were not routinely screened because screening was not part of the infection strategy of the Alrijne hospital. This group of patients are further indicated as the control group.

\section{Intervention}

Patients in the intervention group were screened for nasal $S$. aureus carriage, determined by the nasal swab culture preoperatively. The screening was performed using a dry sterile swab, which was rotated in each nostril. The swab was placed in the WASP (Walk Away Specimen 
Processor, Copan). At the WASP, a blood and mannitol salt agar were inoculated and incubated 48 hours at $35^{\circ} \mathrm{C}$. Strains that were suspected for $S$. aureus were identified in the MALDI-TOF MS (Matrix Assisted Laser Desorption/Ionisation and Time-Of Flight MassaSpectrometrie, Microflex LT, Bruker Daltonik GmbH).

Within 5 days, all patients received the result of their test. Patients with a positive culture for S. aureus were instructed to apply mupirocin (Bactroban ${ }^{\circledR}$, GlaxoSmithKline) ointment 20 $\mathrm{mg} / \mathrm{g}$ three times a day in each nostril for 5 consecutive days starting 6 days before the surgery. Additionally, these patients showered with chlorhexidine gluconate (HiBiScrub®, SSL Healthcare Nederland BV) $40 \mathrm{mg} / \mathrm{ml}$ respectively 1 and 5 days before surgery including the hair.

All patients that received eradication therapy completed the full protocol. Patients with positive cultures for $S$. aureus were screened postoperatively till January 2016 to evaluate the efficiency of our eradication protocol. The postoperatively screening took place at the first appointment with the surgeon after operation (Figure 1). 
Figure 1:Screening and treating schedule for patients $S$. aureus nasal carriage

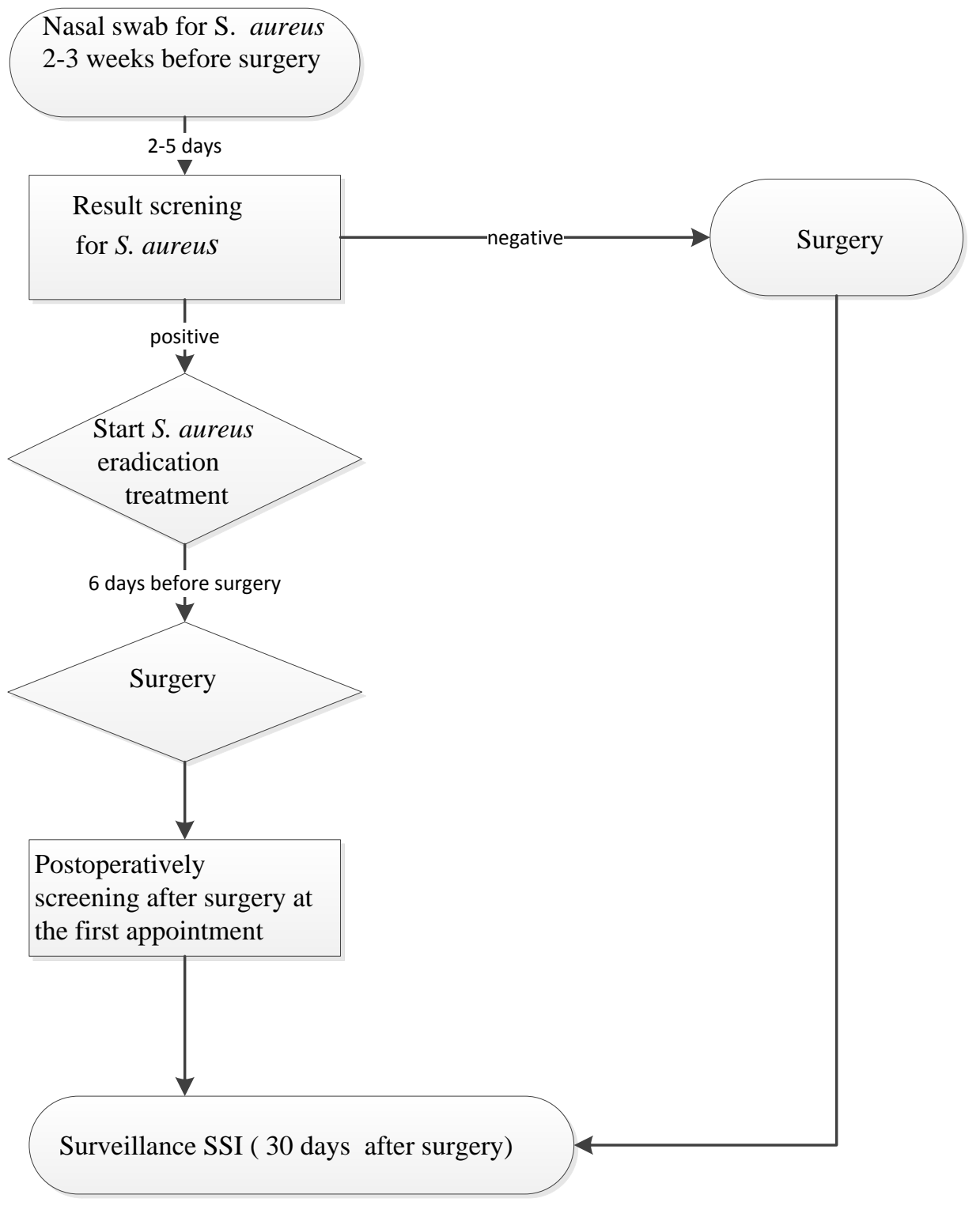

\section{Outcome}

Patients' characteristics such as gender, age and the use of implant were assessed as defined by American Society of Anesthesiology (ASA). ${ }^{8}$ If a wound was collected, the cultured microorganism(s) were documented.

SSI after the initial procedure at 30 days was defined based on PREZIES criteria. ${ }^{2}$

Each SSI was characterized as superficial or deep. A superficial SSI involves only skin or subcutaneous tissue of the incision and at least 1 of the following: (1) purulent drainage from the incision with or without laboratory confirmation; (2) organisms isolated from an aseptically obtained culture of fluid or tissue from the superficial incision; (3) at least 1 of the following signs or symptoms of infection: pain or tenderness, localized swelling, redness, or heat; and deliberate opening of superficial incision by surgeon, unless the incision is culturenegative. A deep SSI involves deep tissues and at least 1 of the following: (1) purulent 
drainage from the incision; (2) the incision spontaneously dehisces or is deliberately opened by a surgeon when the patient has at least 1 of the following signs or symptoms: temperature higher than $38^{\circ} \mathrm{C}$ or localized pain or tenderness, unless site is culture-negative; (3) an abscess or other evidence of infection involving the incision is found on direct examination, during reoperation, or by histopathologic or radiologic examination.

Patients with positive cultures for $S$. aureus were screened postoperatively to evaluate efficiency of the eradication protocol. To determine whether S. aureus eradication was associated with a decreased risk of SSI, the number needed to treat was calculated. Patients were examined routinely by a physician assistant. Signs of SSI were documented on a case report form (CRF). All patients and infections were discussed and confirmed by a surgeon and an infection prevention controller.

\section{Statistics}

The characteristics of the study sample by group (control and intervention) were presented as means and standard deviations for continuous variables, and as percentages for ordinal variables. For group comparisons, continuous variables were analysed with an independent Student's t-test and with a chi-square test for nominal data.

To determine whether $S$. aureus eradication was associated with a decreased risk of SSI descriptive statistics, including frequencies for the independent, variables were included.

Continuous data are presented as means. Categorical data are presented as counts and percentages. Pearson's chi-square and Fisher's exact tests were used for categorical variables and $t$ tests were used for continuous independent variables. In all analyses, statistical under certainties were quantified using corresponding 2-sided 95\% CIs and a P-value of $<0.05$ was considered significant. Statistical analyses were performed with SPSS for Windows, release 21.0 (SPSS Inc., Chicago, IL, USA).

\section{RESULTS AND DISCUSSION}

\section{Study population}

In total, 1543 patients were included for the study in the Alrijne hospital. Of this 1543 patients, 1071 (69.4\%) were screened for S. aureus nasal carriage (intervention group) and $472(30.6 \%)$ patients were not screened for S. aureus nasal carriage (control group). In the intervention group, 221 patients (20.6\%) turned out to be $S$. aureus carriers as at the time of screening, of which two patients screened positive for MRSA (Methicillin Resistant $S$. aureus). All patients were subsequently treated. On January 2016, we stopped screening postoperatively because patients (including the MRSA patients) who were screened a second time postoperatively "remained" clean.

\section{Patient characteristics}


In the baseline characteristics, there were no significant differences between the intervention and the control group (Table 1).

Table 1: Baseline characteristics of 1543 study patients*

\begin{tabular}{llll}
\hline Characteristics & $\begin{array}{l}\text { Screened } \\
(\mathrm{n}=1071)\end{array}$ & $\begin{array}{l}\text { Not screened } \\
(\mathrm{n}=472)\end{array}$ & P value \\
\hline Mean (SD) age & $64(12.2)$ & $64.5(13.3)$ & 0.218 \\
Seks,Male/Female(\% female) & $8 / 1063(99)$ & $4 / 468(99)$ & 1.000 \\
Implant used n(\%) & $102(9.5)$ & $59(13)$ & 0.078 \\
ASA (\%) & & & \\
1 & $227(21.2)$ & $158(33.5)$ & 0.788 \\
2 & $720(67.2)$ & $233(49.4)$ & 0.723 \\
3 & $74(6.9)$ & $41(8.7)$ & 0.934 \\
4 & $2(0.2)$ & $1(0.2)$ & \\
unknown & $48(4.5)$ & $39(8.3)$ & \\
\hline
\end{tabular}

\section{Infection rates}

* April $1^{\text {st }} 2009$ until December $31^{\text {st }} 2016$

The total SSIs were $20(1.8 \%)$ in the intervention group and $12(2.5 \%)$ in the control group $(\mathrm{p}=0.392)$. Of these SSIs, $12(1.1 \%)$ wound swabs tested positive for $S$. aureus in the intervention group versus $12(2.5 \%)$ in the control group $(\mathrm{p}=0.038)$. All SSIs with S. aureus were deep-seated (Table 2).

Table 2: Infection rates in the intervention and control group

\begin{tabular}{|c|c|c|c|c|}
\hline & $\begin{array}{l}\text { Screened } \\
(n=1071)\end{array}$ & $\begin{array}{l}\text { Not screened } \\
(\mathrm{n}=\mathbf{4 7 2})\end{array}$ & $\mathbf{R R}$ & 95\% CI; P value \\
\hline Total SSI (\%) & $20(1.9)$ & $12(2.5)$ & 0.48 & $0.354-1.505 ; \mathrm{P}=0.391$ \\
\hline $\begin{array}{c}\text { Non S. aureus SSI (\%) } \\
\text { Type }\end{array}$ & $8(0.75)$ & $0(0,0)$ & & \\
\hline aerobic and anaerobic flora & 2 & & & \\
\hline Haemolyticus streptococcus $G$ & 1 & & & \\
\hline Streptococcus oralis & 1 & & & \\
\hline E.coli & 1 & & & \\
\hline Staphylococcus capitis & & 1 & & \\
\hline Unable to obtain culture & 2 & & & \\
\hline S. aureus SSI (\%) & $12(1,1)$ & $12(2,5)$ & & $0.194-0.974 ; \mathrm{P}=0.038$ \\
\hline
\end{tabular}

* April $1^{\text {st }} 2009$ until December $31^{\text {st }} 2016$

Sub analysis in the intervention group showed that 4 (1.8\%) patients who screened positive for $S$. aureus, despite treatment and screening negative postoperatively, developed a SSI with S. aureus. It also shows that 8 patients $(0.9 \%)$ who screened negative postoperatively for $S$. aureus also developed a SSI with S. aureus (Table 4).

In this study, all strains of $S$. aureus were sensitive for mupirocin. No serious side effects of either mupirocin and chlorhexidine gluconate were encountered and most importantly, the eradication strategy did not postpone further treatment in any way. 


\section{DISCUSSION}

This prospective cohort study showed that surgical site infections in breast cancer surgery are a frequent and serious complication and most of the SSIs are caused by S. aureus (75\%). The relationship in occurrence of SSI with S. aureus between screening and treating patients of $S$. aureus nasal carriage and patients who were not screened of $S$. aureus nasal carriage is significant (Table 2).

Some authors opted for a treat-all strategy instead of the screen-and-treat strategy that used in this study. ${ }^{9}$ The rationale for treating all patients without screening is reduced cost compared to screen and treat. According to our result the number patient needed to treat was 76 . While treating all patients and omitting the screening process is a lot cheaper than the screen-andtreat method, the treatall strategy is associated with the probability of developing bacterial resistance. $^{10}$

Although, not included in the scope of this study, it was striking to see patients who screened positive preoperatively, were treated and screened negative postoperatively and patients who screened negative preoperatively, develop a surgical site infection with $S$. aureus (Table 3).

Table 3: Sub analysis infection rates in the intervention group*

\begin{tabular}{|c|c|c|c|}
\hline & $\begin{array}{l}\text { Screened } \\
\text { positive }(=221)\end{array}$ & $\begin{array}{l}\text { Screened } \\
\text { negative }(n=850)\end{array}$ & 95\% CI; P-value \\
\hline Total SSI (\%) & $6(2.7)$ & $14(1.6)$ & $0.633-4.387 ; \mathrm{P}=0.275$ \\
\hline Non S. aureus SSI (\%) & $2(0,9)$ & $6(0.7)$ & \\
\hline Type & & & \\
\hline aerobic and anaerobic flora & 1 & 1 & \\
\hline Haemolyticus streptococcus $G$ & & 1 & \\
\hline Streptococcus oralis & & 1 & \\
\hline Staphylococcus capitis & & 1 & \\
\hline Unable to obtain culture & & 2 & \\
\hline Escherichia coli & 1 & & \\
\hline S. aureus SSI (\%) & $4(1.8)$ & $8(0.9)$ & $0.579-6.503 ; \mathrm{P}=0.283$ \\
\hline
\end{tabular}

The cause of these surgical SSIs may be exogenous and several studies show that microorganisms can originate from the operation room (OR) environment ${ }^{11-12-13-14}$ however, Gabriel Birgand et al. reported that the impact of operating-room behaviours on the risk of infection were limited and heterogeneous. ${ }^{15}$

This study has several limitations. Baselines characteristics as diabetes mellitus, smoking and Body Mass Index are not taken in account in this study. These baselines are associated with higher risks of SSI outcomes ${ }^{16-17}$ and may have influenced the development of a SSI in this study. 


\section{CONCLUSION}

In conclusion, this study shows that our screen-and-treat strategy prescribing mupirocin nasal ointment and chlorhexidine soap for patients requiring breast surgery due to cancer with positive nasal swab cultures for S. aureus, resulted in significantly less SSIs with S. aureus. We recommend this strategy to reduce complications arising from SSIs with S. aureus in breast cancer surgery, because additional advantages are lack of major side effects, treatment did not delay surgery and might saves both life years and medical costs at the same time. However, more research is needed to investigate if there is a possible correlation between $S$. aureus originating from the environment and the risk of SSI during breast cancer surgery, which could lead to a further reduction in SSIs with S. aureus.

\section{REFERENCES}

1. www.cbs.nl

2. https://www.rivm.nl/Onderwerpen/P/PREZIES/Incidentieonderzoek_POWI/Protocol_ module_Postoperatieve_wondinfecties/Protocollen_Postoperatieve_Wondinfecties_ar chief

3. Urban JA:Cost analysis of surgical site infections. Surg Infect (Larchmt). 2006; 7 Suppl1:S19-22.

4. Jones DJ, rBunn F, Bell-Syer SV: Prophylactic antibiotics to prevent surgical site infection after breast cancer surgery. Cochrane Database of Systematic Reviews 2014, Issue 3. Art.No.:CD005360. DOI:10.1002/14651858.CD005360.pub4.

5.Bode LG, Kluytmans JA, Wertheim HF, Bogaers D, Vandenbroucke-Grauls CM, Roosendaal R. et al. Preventing surgical-site infections in nasal carriers of Staphylococcus aureus. N Engl J Med. 2010;362(1):9-17.

6. Kluytmans J, Van Belkum A, Verbrugh H (1997) Nasal carriage of Staphylococcus aureus: epidemiology, underlying mechanisms, and associated risks. Clin Microbiol Rev10(3): 505-20. 6.

7. https://www.rivm.nl/Onderwerpen/P/PREZIES/Incidentieonderzoek_POWI/Referenti ecijfers_POWI

8. http://asahq.org/

9. Marjan W. M. Wassenberg, G. Ardine de Wit, Marc J. M. Bonte. Cost-Effectiveness of Preoperative Screening and Eradication of Staphylococcus aureus Carriage. PLoS ONE | www.plosone.org May 2011 | Volume 6 | Issue 5 | e14815.

10. Cookson BD. The emergence of mupirocin resistance: a challenge to infection control 11. and antibiotic prescribing practice. J Antimicrob Chemother. 1998; 41(1):11-8. PMID: 9511032. 
12. L. Silvia Munoz-Price, David J. Birnbach, David A. Lubarsky, Kristopher L. Arheart EdD, Yovanit Fajardo-Aquino, Mara Rosalsky, Timothy Cleary, Dennise DePascale , Gabriel Coro, Nicholas Namias and Philip Carling. Decreasing Operating Room Environmental Pathogen Contamination through Improved Cleaning Practice. Infection Control and Hospital Epidemiology Vol. 33, No. 9 (September 2012), pp. 897-904.

13. Terri Link, Catherine Kleiner, Mary P. Mancuso, Oliwier Dziadkowiec, Katherine Halverson-Carpenter. Determining high touch areas in the operating room with levels of contamination. American Journal of Infection Control 44 (2016) 1350-5.

14. H. Brohus, K. D. Balling, D. Jeppesen. Influence of movements on contaminant transport in an operating room. Indoor Air 2006; 16: 356-372.

15. Stephanie Thomas, Rish Palmer, Edward Phillipo, Geoffrey Chipungu. Reducing bacterial contamination in an Orthopedic Theatre ventilated by natural ventilation, in a Developing Country.J Infect Dev Ctries 2016;10(5):518-522. doi:10.3855/jidc.7436.

16. Gabriel Birgand, Christine Azevedo, Gaelle Toupet, Roger Pissard-Gibollet, Bruno Grandbastien, Eric Fleury, Jean-Christophe Lucet. Attitudes, risk of infection and behaviours in the operating room (the ARIBO Project): a prospective, cross-sectional study. BMJ Open 2014;4:e004274. doi:10.1136/bmjopen-2013-004274.

17. E. Tartari, V. Weterings, P. Gastmeier, J. Rodríguez Baño, A. Widmer, J. Kluytmans and Voss. Patient engagement with surgical site infection prevention: an expert panel Perspective. Antimicrobial Resistance and Infection Control (2017) 6:45DOI 10.1186/s13756-017-0202-3.

18. Liu Y, Dong Y, Wu X, Chen H, Wang S. Influence of high body mass index on mortality and infectious outcomes in patients who underwent open gastrointestinal surgery: A meta-analysis. Am J Infect Control. 2016 May 1;44(5):572-8. doi: 10.1016/j.ajic.2015.12.009. Epub 2016 Jan 29.

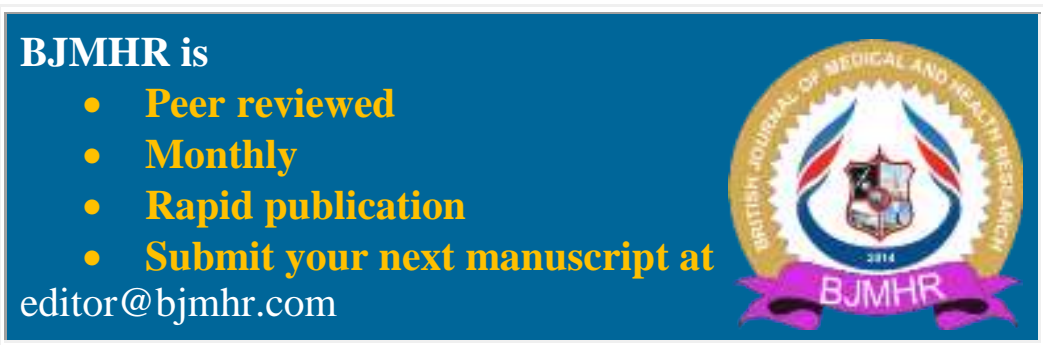

